\title{
British Rhinological Society Consensus Guidance on the use of biological therapies for Chronic Rhinosinusitis with Nasal Polyps
}

\author{
Claire Hopkins ${ }^{1}$, Jo-Lyn McKenzie ${ }^{2}$, Shahram Anari ${ }^{3}$, Sean Carrie ${ }^{4}$, Yujay Ramakrishnan ${ }^{5}$, \\ Naveed Kara ${ }^{6}$, Carl Philpott ${ }^{7}$, Jonathan Hobson ${ }^{8}$, Ali Qureishi ${ }^{9}$, Benjamin Stew ${ }^{10}$, Philip \\ Harries $^{11}$, Simon Gane ${ }^{12}$, Raj Bhalla ${ }^{13}$, Abigail Walker ${ }^{14}$, Iain Hathorn ${ }^{15}$, and Valerie \\ Lund $^{16}$ \\ ${ }^{1}$ Guy's and St Thomas' Hospitals NHS Trust \\ ${ }^{2}$ Royal Brisbane and Women's Hospital \\ ${ }^{3}$ University Hospitals Birmingham NHS Foundation Trust \\ ${ }^{4}$ Freeman Hospital \\ ${ }^{5}$ Nottingham University Hospitals NHS Trust \\ ${ }^{6}$ County Durham \& Darlington NHS Foundation Trust \\ ${ }^{7}$ University of East Anglia \\ ${ }^{8}$ Manchester University NHS Foundation Trust \\ ${ }^{9}$ Oxford University Hospitals NHS Foundation Trust \\ ${ }^{10}$ University Hospital of Wales Healthcare NHS Trust \\ ${ }^{11}$ University Hospital Southampton NHS Foundation Trust \\ ${ }^{12}$ Royal National Ear, Nose and Throat and Eastman Dental Hospital \\ ${ }^{13}$ Central Manchester \& Manchester Children's Foundation Trust \\ ${ }^{14}$ St Georges' Hospital \\ ${ }^{15}$ University of Edinburgh Division of Medical and Radiological Sciences \\ ${ }^{16}$ Royal National Throat Nose and Ear Hospital
}

February 28, 2021

\begin{abstract}
Objectives We set out to create Consensus Guidelines, based on current evidence and relative risks of adverse effects and the costs of different treatments, that reflect the views of the British Rhinological Society (BRS) Council on where the use of biologics should be positioned within treatment pathways for CRSwNP, specifically in the setting of the National Health Service (NHS). Methods An expert panel of 16 members was assembled. A review of the literature and evidence synthesis was undertaken and circulated to the panel We used the RAND/UCLA methodology with a multi-step process to make recommendations on the use of biologics. Setting and participants N/A Results Recommendations were made, based on underlying disease severity, prior treatments and co-morbidities. A group of patients for whom biologics were considered an appropriate treatment option for CRSwNP was defined. Conclusions Although biologics are not currently available for the treatment of CRSwNP, the BRS Council have defined a group of patients who have higher rates of 'failure' with current treatment pathways, higher resource use and are more likely to suffer with uncontrolled symptoms. We would urge NICE to consider approval of biologics for such indications without applying further restrictions on use.
\end{abstract}

British Rhinological Society Consensus Guidance on the use of biological therapies for Chronic Rhinosinusitis with Nasal Polyps 


\section{Abstract \\ Objectives}

We set out to create Consensus Guidelines, based on current evidence and relative risks of adverse effects and the costs of different treatments, that reflect the views of the British Rhinological Society (BRS) Council on where the use of biologics should be positioned within treatment pathways for CRSwNP, specifically in the setting of the National Health Service (NHS).

Design

An expert panel of 16 members was assembled. A review of the literature and evidence synthesis was undertaken and circulated to the panel We used the RAND/UCLA methodology with a multi-step process to make recommendations on the use of biologics.

Setting N/A

Participants N/A

Results

Recommendations were made, based on underlying disease severity, prior treatments and co-morbidities. A group of patients for whom biologics were considered an appropriate treatment option for CRSwNP was defined.

Conclusions

Although biologics are not currently available for the treatment of CRSwNP, the BRS Council have defined a group of patients who have higher rates of 'failure' with current treatment pathways, higher resource use and are more likely to suffer with uncontrolled symptoms. We would urge NICE to consider approval of biologics for such indications without applying further restrictions on use.

\section{Introduction}

Chronic rhinosinusitis with nasal polyps (CRSwNP) is a prevalent condition characterised by a significant impact on quality of life and productivity, with frequent recurrence after both medical and surgical treatment(1). Oral corticosteroids are effective at achieving short term symptom improvement $(2,3)$, but benefit is limited due to the risk of side effects which accrue with repeated use (4). Surgery has been shown to achieve more durable symptomatic benefits (5), but is associated with pain and discomfort, time for recovery, a small but definite risk of serious complications(6), and, based on previous data, more than one in 5 patients will require another surgery within 5 years of follow-up(5). Poor disease control is commonplace, driving the search for alternative treatment strategies.

Monoclonal antibodies (Mabs) are biological treatments that target specific inflammatory mediators or immune cells. Growing understanding of the pathophysiological pathways found in CRSwNP and evidence of efficacy in similar patterns of Type 2 inflammation found in asthma and atopic dermatitis have led to trials evaluating use in CRSwNP. As such therapies are evaluated and are shown to be effective, it will be essential to consider where they should be placed in current treatment pathways for CRSwNP, particularly with respect to the use of corticosteroids and surgeries.

We set out to create Consensus Guidelines, based on current evidence and relative risks of adverse effects and the costs of different treatments, that reflect the views of the British Rhinological Society (BRS) Council on where the use of biologics should be positioned within treatment pathways for CRSwNP, specifically in the setting of the National Health Service (NHS). These guidelines were created in early 2021 and it is anticipated that they will evolve as further evidence accrues and the relative costs and availability of treatments change.

\section{Methods}

\section{Expert panel}


An expert panel of 16 members was assembled. All BRS Council members (elected from all parts of the United Kingdom) were invited to participate, but could decline based on lack of availability, limited knowledge or experience within the area of specialist interest, or if working outside the NHS setting. Additional panellists were invited who had expertise in this area.

\section{Literature review and evidence summary}

A background literature review and synthesis of the evidence was undertaken by a core group of the panel, and key evidence summarised (see Appendix 1). A recently updated version of the Cochrane review, 'Biologics for chronic rhinosinusitis' was circulated in full, along with another recent systematic review of use in CRSwNP(7) and two previously published guidelines on indications for use of biologics, from EPOS(8) and EUFOREA(9). Key outcomes from relevant trials were summarised and circulated in data tables (Appendix 2).

\section{Indications for consideration}

The core group defined what symptoms, past medical history and relevant diagnostic tests might influence the decision regarding whether biologic prescription might be appropriate. Their responses were used to form a series of matrixes that categorise patients according to these key indications. Specific combinations of associated comorbidities and previous treatment were considered, alongside wider statements concerning the delivery of biologics. One hundred clinical scenarios were considered, stratified by the presence of comorbid asthma, N-ERD or AFRS, the number of previous surgeries (or if a patient was unfit surgery), and the use of INCS or OCS (or if a patient was unable to use them). Other characteristics such as baseline symptom scores, the importance of radiological imaging and the extent of disease on radiological imaging were also considered.

Consensus process We used the RAND/UCLA methodology with a multi-step process (www.rand.org)(10). Our expert panel undertook a 2 round modified Delphi process of ranking and classifying appropriateness of different investigations and treatment options. Using a 9-point Linkert scale, for each indication the panellists scored whether a treatment was either:

- Not recommended/ Inappropriate; should not be prescribed for the indication described within the NHS, based on current evidence base and costs (scored 1 to 3 ),

- Uncertain; (scored 4 to 6 ),

- Recommended / Appropriate; should be prescribed for the indication described within the NHS, based on current evidence base and costs (scored 7 to 9)

Free text comments were encouraged if greater context was required, if the question was ambiguous or if anything had been overlooked.

The final recommendation was based on the median ranking scores collated from each clinical scenario provided that there was consensus. Consensus was defined as the requirement for more than $70 \%$ of responses to fall into the category defined by the median, and when this score fell in either recommended or not recommended, less than $15 \%$ of responses were scored as the opposite.

The scores at the end of round 1 were analysed and presented back to the group, with the distribution of scores summarised for each question. The panel was then asked to repeat the scoring for any items where consensus has not been reached having considered if they wished to revise their previous score.

Round 2 scores were evaluated in the same way. When the median fell between 4-6, or if the median fell in either 1-3 or 6-9 and the definition of consensus was not met, no recommendation was made, and the use of biologics for the given indication was considered uncertain.

\section{Results}

Sixteen panellists completed the first round, and 15 completed the second round. 
Of the 100 clinical scenarios, consensus was reached on 77 (36 at round 1, increasing to 77 after round 2). Of the remaining 23 scenarios, the median fell between 4-6, or if the median fell in either 1-3 or 6-9 and the definition of consensus was not met, no recommendation was made.

\section{Recommendations}

The recommendations are summarised in figure $1 \mathrm{a}$ and $1 \mathrm{~b}$

Figure 1a summarises all situations where agreement was reached that it is appropriate that biologics be considered in the treatment of CRSwNP within the NHS at the current time.

Figure 1b summarises all situations where agreement was reached that it is NOT appropriate that biologics be considered in the treatment of CRSwNP within the NHS at the current time.

For all other combinations of OCS, surgery and comorbidity which are not included in either figure, agreement was not reached. In almost all remaining scenarios, the median answer of the was 'uncertain' and there was no move towards consensus between rounds.

More specifically;

In all patients, regardless of associated comorbidity,

Patients should have at least moderate symptoms measured on the SNOT-22 (>20) or VAS scale (>3) on current treatment, and a Lund-Mackay score of 8 or more to be considered for a biologic

Biologics should NOT be considered if the patient has not used corticosteroids in any form in the preceding 12 months.

Biologics should be considered if patients have received 2 or more courses of OCS, or are unable to take OCS AND have had 3 or more surgeries, or are considered unfit for surgery (under local or general anaesthetic)

In patients without asthma or N-ERD; in addition to the criteria above for all patients

Biologics should NOT be considered if the patient has not used oral corticosteroids in the preceding 12 months.

Biologics should NOT be considered if the patient has not had previous surgery

Biologics should NOT be considered if the patient has required 1 - 2 courses of oral corticosteroids in the preceding 12 months AND has not undergone previous surgery or has undergone one previous surgery.

In patients with asthma but no $N$-ERD, in addition to the criteria for all patients

Biologics should NOT be considered if the patient has not had previous surgery

Biologics should NOT be considered if the patient has not used oral corticosteroids in the preceding 12 months AND has undergone 2 or fewer previous surgeries

Biologics should be considered if patients have required at least 1 course of steroids in the last year or are unable to take OCS AND have had 3 or more previous surgeries or are unfit for surgery

Biologics should be considered if patients have received 2 or more courses of OCS, or are unable to take OCS AND have had 2 or more surgeries, or are considered unfit for surgery (under local or general anaesthetic)

In patients with asthma and $N$-ERD, in addition to the criteria above for all patients

Biologics should NOT be considered if the patient has not had previous surgery AND have not received more than 2 courses of OCS

Biologics should NOT be considered if the patient has not used oral corticosteroids in the preceding 12 months AND has undergone 1 or fewer previous surgeries 
Biologics should be considered if patients have required at least 1 course of steroids in the last year or are unable to take OCS AND have had 2 or more previous surgeries or are unfit for surgery

Biologics should be considered if patients have received 2 or more courses of OCS, or are unable to take OCS AND have had 1 or more surgeries, or are considered unfit for surgery (under local or general anaesthetic)

In patients with AFRS

It was noted that at the current time there is insufficient evidence to support effectiveness, and therefore trials must demonstrate that biologics are effective in this cohort and to determine whether this requires surgery to be performed in parallel. However, providing these criteria are met by future research, the following conditional recommendations could be made;

Biologics should NOT be considered if the patient not had previous surgery

Biologics should NOT be considered if the patient has not used oral corticosteroids in the preceding 12 months

Biologics should NOT be considered if the patient has required $1-2$ courses of oral corticosteroids in the preceding 12 months AND has not undergone previous surgery or has undergone one previous surgery.

Biologics should be considered if patients have required at least 1 course of steroids in the last year or are unable to take OCS AND have had 3 or more previous surgeries or are unfit for surgery

Biologics should be considered if patients have received 2 or more courses of OCS, or are unable to take OCS AND have had 2 or more surgeries, or are considered unfit for surgery (under local or general anaesthetic)

\section{General recommendations on the provision of biologics in an ENT setting}

Biologics should be available only in a designated specialist centres that can offer appropriate alternative interventions

Specialist centres should be co-located with Severe Asthma clinics that are already providing biologic therapies.

A CT scan is required to establish extent of disease and that of previous surgery

Endoscopic confirmation is essential in the assessment of eligibility and response

Revision surgery should be considered prior to a biologic if the previous surgery was a polypectomy without opening the sinuses

Until there is evidence to support the safety and efficacy of biologics in CRS without nasal polyps, biologics should NOT be prescribed

Outside of the NHS, self-paying patients with CRSwNP may select biologic therapies as an alternative to surgery or OCS provided that they have been given information regarding the risks and benefits of each option

\section{Discussion}

The panel have made a series of recommendations which define a group of patients in whom the use of biologics is considered appropriate in the treatment of CRSwNP, and a group where use would be considered inappropriate, considering the current evidence base, costs and capacity in the setting of the NHS.

The RAND/UCLA appropriateness methodology is well described and has previously been utilised defining appropriateness criteria for ESS during management of uncomplicated adult CRS and adult recurrent acute rhinosinusitis $(1,11,12)$. We have used a similar process to develop BRS guidance on the management of incidental findings in the maxillary sinuses with regard to dental implantation(13), and to develop guidance on the treatment of COVID-19 related loss of smell(14). This exercise or process aims to detect and achieve consensus amongst a group of experts, and is ideally suited to evaluating the appropriateness of use of 
medical interventions where the evidence base is limited, which consume significant resources or where use remains controversial. Defining appropriate use of biologics in CRSwNP would therefore seem to be well suited to the methodology.

Biologic therapy using Mabs that block the action of interleukins or other targets central to type 2 inflammation now play an important role in the management of difficult-to-treat asthma and many of these treatments have also been shown to be effective in the management of severe CRSwNP. Dupilumab, an anti IL4/13 receptor mab, and omalizumab, an anti-IgE mab, have been shown to achieve significant reductions in polyp size and nasal congestion in large phase 3 studies $(15,16)$. Both have now been granted FDA and EMA approval for use in patients with CRSwNP, and are currently available for use in the US and selected European countries. Other drugs will likely soon follow, specifically with phase 3 trials completed for mepolizumab(17) and benralizumab, which target IL5.

Although biologicals have been shown to reduce the need for surgical intervention for CRSwNP(17), their high costs and the need for long term treatment mean that this is unlikely to be the most cost-effective treatment across the whole population with CRSwNP, even if superior in terms of long-term symptom control in the difficult-to-treat group. Scangas et al undertook a Markov decision tree cost-effectiveness model over 20 years(18), and found, based on US costs (which may not be applicable in all healthcare setting), that a strategy of sinus surgery cost circa $\$ 50,000$ producing 9.80 QALYs while dupilumab treatment costs $\$ 535,000$ but produced 8.95 QALYS. Surgery was more cost-effective regardless of the frequency of revision surgery. Similarly, in asthma, although the efficacy of biologic therapy is well established, none of the currently available drugs have been found to be cost-effective(19).

Currently, no biologic treatments have been approved by NICE for the treatment of patients with CRSwNP. NICE considers evidence of effectiveness of new interventions, but applies a standard threshold range with an upper limit of $£ 20-30,000$ per QALY and a budget impact test, where drugs that cost more than £20million in any one of their first three years of use trigger commercial discussions to mitigate the impact on the wider NHS, or further restrictions on usage (www.nice.org.uk). Using the data from Scangas et al, with a $£ 43,500$ cost per QALY at current currency conversion rates, it is unlikely that the NICE threshold could be met if biologics were prescribed to all patients with CRSwNP.

However, the panel's recommendations identify a group who are more likely to fail to achieve long term benefit from conventional treatment pathways and to undergo repeated interventions with higher associated healthcare resource utilisation. A recent study has shown that patients with a history of previous surgery are twice as likely to need further revision surgery, or those with a history of N-ERD five times more likely to require further revision when undergoing endoscopic sinus surgery(20). Therefore, as the patients identified by the panel have higher direct costs over their lifetime than other patients with CRSwNP, use of biologics in this subgroup are more likely to be cost-effective. Patients with higher rates of revision surgery also likely derive less symptomatic benefit from conventional treatment pathways; indeed a recent study found that $43 \%$ of patients' symptoms were uncontrolled after sinus surgery and this again was more common after revision surgery and in the setting of $\mathrm{N}-\operatorname{ERD}(21)$. Our criteria will also, therefore, help to identify patients for who current treatments are likely unsuccessful in achieving adequate disease control, and where biologics may offer the only option that may achieve long term disease control. We, therefore, hope that NICE and other organisations will consider our criteria to define a group for whom biologics should be approved even if the usual threshold limit per QALY cannot be met, and not seek to impose further restrictions on usage over and above those defined by the panel (Fig 1).

The criteria used by the panel in reaching consensus where biologics are considered appropriate define a smaller group of eligible patients than either the EPOS(8) or EUFOREA(9) criteria for patient selection for biologic therapy. The scenarios where the panel felt that use of a biologic was uncertain included patients with at least one previous surgery, many of whom would meet the EPOS criteria for biologics, although it should be noted that the BRS set a lower threshold of symptom severity at baseline, measured using the SNOT-22 or VAS. Many of the scenarios where use of biologics in the NHS is currently considered 'uncertain' by the BRS panel represent patients who would have been eligible for recruitment to published trials demonstrating 
the effectiveness of biologics, and within the trials achieved significant improvements in nasal polyp score and quality of life on biologics. Therefore, it is important to state that the BRS recommendations are not intended to predict response to treatment to biologics, but only to define a group where the panel felt use was appropriate given the financial restrictions within the NHS at the current time. It is very likely that recommendations for the scenarios rated 'uncertain' will change if the relative cost of treatment is reduced in the future.

Panellists recommended that biologic treatments for CRSwNP, if approved for use within the NHS, should be delivered within centres of excellence co-located with difficult to treat asthma, where there is pre-existing experience of treatment with biologics. Centres should also be able to offer a range of other treatments. This will also have the likely impact that patients with difficult to treat CRSwNP, for example those with $\mathrm{N}$-ERD, are directed to specialist centres that can ensure that surgery is performed optimally and that patients are considered for adjunctive treatments such as post-operative desensitisation, thus reducing the need for biologics. Indeed, a recent paper has shown that more extensive surgery in patients with CRSwNP was associated with lower rates of revision $\operatorname{surgery}(20)$; creation of specialist centres will, therefore, likely improve outcomes from other interventions such as sinus surgery. Panellists commented that in future, the extent of previous surgery and the interval between previous surgery may also be considered in the decision making process, and perhaps patients who have only had more limited surgery be considered for revision surgery before biological therapies.

While the panel made recommendations for use in AFRS, it was noted that clear evidence of efficacy on trials was needed before these should be implemented. The panel also agreed that there was no indication for use in patients with CRS without nasal polyps in the absence of evidence in this group

Once biologic therapies are initiated, the response to treatment must be assessed to determine if a patient should continue treatment; how this should be determined is beyond the remit of the current study. Further limitations of these current recommendations are that biomarkers, such as eosinophil levels in blood or tissue, were not considered in the clinical scenarios, as the panel felt that there is insufficient evidence to determine how these aid selection or predict response to treatment. Biologics were considered as a collective intervention but in reality different biologics, defined by their inflammatory target, differ in terms of costs and effectiveness. The impact of COVID-19 on healthcare delivery in the NHS at the current time was not considered - this may favour biologics over surgery, for example, if demand for surgery greatly exceeds capacity. Finally, we have not included patient preferences for different treatments into our recommendation but these of course play an important role in the final decision making with regards to use if biologics become available within the UK.

\section{Conclusions}

Using a modified Delphi technique, we have defined a patient cohort with CRSwNP where the BRS Council believe use of biologics are appropriate. This group have higher rates of 'failure' with current treatment pathways, higher resource use and are more likely to suffer with uncontrolled symptoms. We would urge NICE to consider approval of biologics for such indications without applying further restrictions on use.

Ethical approval

There was no need for ethical approval for this study

Data availability

The results of scoring of the Delphi round may be made available to the editorial team on request but it is not common practice to publish the scores alongside the recommendations.

\section{References}

1. Fokkens WJ, Lund VJ, Hopkins C, Hellings PW, Kern R, Reitsma S, et al. European Position Paper on Rhinosinusitis and Nasal Polyps 2020. Rhinology. 2020;58(Suppl S29):1-464. 
2. Head K, Chong LY, Hopkins C, Philpott C, Schilder AG, Burton MJ. Short-course oral steroids as an adjunct therapy for chronic rhinosinusitis. Cochrane Database Syst Rev. 2016;4:CD011992.

3. Head K, Chong LY, Hopkins C, Philpott C, Burton MJ, Schilder AG. Short-course oral steroids alone for chronic rhinosinusitis. Cochrane Database Syst Rev. 2016;4:CD011991.

4. Price D, Castro M, Bourdin A, Fucile S, Altman P. Short-course systemic corticosteroids in asthma: striking the balance between efficacy and safety. European respiratory review : an official journal of the European Respiratory Society. 2020;29(155).

5. Hopkins C, Slack R, Lund V, Brown P, Copley L, Browne J. Long-term outcomes from the English national comparative audit of surgery for nasal polyposis and chronic rhinosinusitis. Laryngoscope. 2009;119(12):245965.

6. Hopkins C, Browne JP, Slack R, Lund VJ, Topham J, Reeves BC, et al. Complications of surgery for nasal polyposis and chronic rhinosinusitis: the results of a national audit in England and Wales. Laryngoscope. 2006;116(8):1494-9.

7. Kim C, Han J, Wu T, Bachert C, Fokkens W, Hellings P, et al. Role of Biologics in Chronic Rhinosinusitis With Nasal Polyposis: State of the Art Review. Otolaryngol Head Neck Surg. 2021;164(1):57-66.

8. Fokkens WJ, Lund VJ, Hopkins C, Hellings PW, Kern R, Reitsma S, et al. Executive summary of EPOS 2020 including integrated care pathways. Rhinology. 2020;58(2):82-111.

9. Fokkens WJ, Lund V, Bachert C, Mullol J, Bjermer L, Bousquet J, et al. EUFOREA consensus on biologics for CRSwNP with or without asthma. Allergy. 2019;74(12):2312-9.

10. Fitch K BS, Aguilar MD, Burnand B, LaCalle JR. The RAND/UCLA appropriateness method user's manual. RAND CORP SANTA MONICA CA;2001.

11. Fitch K, Bernstein SJ, Aguilar MD, Burnand B, LaCalle JR. The RAND/UCLA appropriateness method user's manual. RAND CORP SANTA MONICA CA; 2001.

12. Rudmik L, Beswick DM, Alt JA, Bhattacharyya N, Chester AC, Gray ST, et al. Appropriateness Criteria for Surgery in the Management of Adult Recurrent Acute Rhinosinusitis. The Laryngoscope. 2019;129(1):3744.

13. Amin N, Walker A, Alobid I, Anari S, Bast F, Bhalla RK, et al. Defining appropriateness criteria for endoscopic sinus surgery in the management of adult dental implant patients with incidental maxillary sinus findings on conebeam computed tomography. Clin Otolaryngol. 2020.

14. Hopkins C, Alanin M, Philpott C, Harries P, Whitcroft K, Qureishi A, et al. Management of new onset loss of sense of smell during the COVID-19 pandemic - BRS Consensus Guidelines. Clin Otolaryngol. 2021;46(1):16-22.

15. Bachert C, Han JK, Desrosiers M, Hellings PW, Amin N, Lee SE, et al. Efficacy and safety of dupilumab in patients with severe chronic rhinosinusitis with nasal polyps (LIBERTY NP SINUS-24 and LIBERTY NP SINUS-52): results from two multicentre, randomised, double-blind, placebo-controlled, parallel-group phase 3 trials. Lancet. 2019;394(10209):1638-50.

16. Gevaert P, Omachi TA, Corren J, Mullol J, Han J, Lee SE, et al. Efficacy and safety of omalizumab in nasal polyposis: 2 randomized phase 3 trials. J Allergy Clin Immunol. 2020;146(3):595-605.

17. Bachert C, Sousa AR, Lund VJ, Scadding GK, Gevaert P, Nasser S, et al. Reduced need for surgery in severe nasal polyposis with mepolizumab: Randomized trial. J Allergy Clin Immunol. 2017;140(4):1024-31 e14.

18. Scangas GA, Wu AW, Ting JY, Metson R, Walgama E, Shrime MG, et al. Cost Utility Analysis of Dupilumab Versus Endoscopic Sinus Surgery for Chronic Rhinosinusitis With Nasal Polyps. Laryngoscope. 
2021;131(1):E26-E33.

19. Tice JA WJ, Synnott P, Kumar V, Adair E, Rind D, et al. Biologic Therapies for Treatment of Asthma Associated with Type 2 Inflammation. Inst Clin Econ Rev. 2018;1-132.

20. Hopkins C, Lund V. Does time from previous surgery predict subsequent treatment failure in Chronic Rhinosinusitis with Nasal Polyps? Rhinology. 2021(In press).

21. van der Veen J, Seys SF, Timmermans M, Levie P, Jorissen M, Fokkens WJ, et al. Real-life study showing uncontrolled rhinosinusitis after sinus surgery in a tertiary referral centre. Allergy. 2017;72(2):282-90.

Legends

Figure 1a Agreed criteria where it was considered appropriate to consider treatment with a biologic

Figure 1b Agreed criteria where it was considered not to be appropriate to consider treatment with a biologic

Biologics SHOULD be considered if the following conditions are met;

Patient with CRS with nasal polyps AND moderate symptom severity or more

(SNOT22 $>=21$ or VAS $>=4$ ) AND Lund-Mackay CT Score $>=8$

AND a score of 5 points or more out of a possible 7;

Number of courses of OCS in last 12 months (to max of 2 points)

1 course in last 12 months $=1$ point

2 or more courses in last 12 months $=2$ points

Unable to take OCS due to medical contraindications $=2$ points

Number of previous surgeries for CRSwNP (to max of 3 points)

1 previous sinus surgery $=1$ point

2 previous sinus surgeries $=2$ points

3 or more previous sinus surgeries $=3$ points

If unfit for surgery $=3$ points

Comorbid asthma $=1$ point

Comorbid N-ERD = 1 point in addition to 1 point for co-morbid asthma 
Biologics SHOULD NOT be considered if any of the following conditions are met;

\section{CRSsNP}

Mild symptom severity (SNOT22 $=<20$ or VAS $=<3$, depending on which measure is used)

Lund-Mackay CT score $<8$

No corticosteroids in any form (INCS or OCS) in preceding 12 months

No OCS in the last 12 months AND and no asthma / NERD

No OCS in the last 12 months AND asthma if 2 or fewer previous surgeries

No previous surgery AND no NERD (regardless of asthma status)

No previous surgery AND NERD if two or fewer courses of OCS

1-2 OCS in the last 12 months AND one or fewer previous surgeries AND no asthma or NERD 